\title{
ACURÁCIA DO TESTE DE PAPANICOLAOU NO DIAGNÓSTICO DE LESÕES PRECURSORAS DO CÂNCER CERVICAL
}

\section{Accuracy of Pap test in diagnosis of precursor lesions of cervical cancer}

Maria Inês Rosa ${ }^{1}$, Priscila Seibert, ${ }^{1}$ Bruno Rosa Silva ${ }^{2}$

1 Laboratório de Epidemiologia da Universidade do Extremo Sul Catarinense Criciúma, SC, Brasil.

2 Programa de Residência em Ginecologia e Obstetrícia, Maternidade Marieta Konder Bornhausen, Itajaí-SC, Brasil.

Suporte financeiro: Universidade do Extremo Sul Catarinense

\section{Endereço para correspondência:}

Maria Inês Rosa

Rua Cruz e Souza, 510, Bairro Pio Correa, Criciúma-SC- Brasil.

Telefone: +55 4834339976 Fax: +55 4834335766

CEP 88811-550

Email: mir@unesc.net 


\title{
Resumo
}

Objetivo: Avaliar a acurácia da citologia e colposcopia. Métodos: Foi realizado um estudo retrospectivo entre 184 mulheres consecutivas que foram submetidas à biópsia cervical. Os desfechos (lesões precursores e câncer cervical) assim como os resultados do teste de Papanicolaou e colposcopia, foram dicotomizadas em lesão intraepitelial de baixo grau ou menos e lesão intraepitelial de alto grau ou mais. Foi calculado sensibilidade, especificidade, valor preditivo positivo (VPP) e negativo (VPN) para cada, todos com intervalo de confiança de 95\%. Resultados: Foram encontrados 184 pacientes que preencheram os critérios do estudo. A média de idade foi de $30.2 \pm 10.6$ (variação 15-89). A sensibilidade, especificidade e acurácia foram melhores na colposcopia do que no exame de Papanicolaou: a sensibilidade foi de 70,4\% (IC 95\% 67,0\% - 81,0\%) e 54,0\% (IC 95\% 46,8\% - 61,2\%) respectivamente, a especificidade foi de $96,3 \%$ (95\% IC 93,0\% -99,0\%) e $91,0 \%$ (IC 95\% 86,9\%-95,1\%) respectivamente, e a acurácia de 88,9\% (IC 95\% 75,3\% $86,6 \%$ ) e $80,9 \%$ (IC $95 \% \quad 75,3 \% \quad-86,6 \%$ ) na colposcopia e citologia, respectivamente. Conclusão: Os falsos negativos foram um grande problema detectado em nosso estudo, eles não diagnosticaram $46 \%$ dos casos de lesão intraepitelial escamosa de alto grau (LIEAG) ou mais pela citologia e $26 \%$ pela colposcopia.

Palavras-chave: Acurácia; Exame de Papanicolaou; Lesões Precursoras; Colposcopia.

\begin{abstract}
Purpose: to evaluate the accuracy of cytology and colposcopy with histopathology results. Methods: We performed a retrospective study among 184 consecutive women who were submitted to cervical biopsy. The outcomes (precursors lesions and cervical cancer), the findings of Pap test and colposcopy, were dichotomized in low-grade squamous intraepithelial lesion or lower and high-grade squamous intraepithelial lesion or more. Was calculated sensitivity, specificity, positive and negative predictive value (PPV, NPV) of each test was computed and all of them have $95 \%$ exact binomial confidence intervals. Results: we found 184 patients who met the criteria of the study. The mean age was $30.2 \pm 10.6$ (range 15-89). The
\end{abstract}


Artigo Original

Atenção à Saúde

sensitivity, specificity and accuracy was better in colposcopy than Pap test: sensitivity was 70.4\% (95\% IC 67.0\% - 81.0\%) and 54.0\% (95\% IC 46,8\% - 61,2\%) respectively, specificity was 96.3\%(95\% IC 93.0\%-99.0\%) and 91.0\% (95\% IC 86.9\%-95.1\%) respectively and accuracy 88,9\% (95\% IC 75.3\%-86.6\%) and 80,9 $\%(95 \%$ IC 75.3\%-86.6\%) in colposcopy and cytology respectively. Conclusion: False negatives were a great problem detected in our study, they did not diagnose $46 \%$ of HSIL cases or more by cytology and $26 \%$ by colposcopy.

Keywords: Pap Test; Accuracy; Precursor Lesions; Colposcopy.

\section{INTRODUÇÃO}

O câncer cervical representa a segunda neoplasia ginecológica mais comum no mundo, com cerca de 510.000 novos casos de câncer diagnosticados anualmente ${ }^{1}$.

A infecção pelo papilomavirus humano (HPV) de alto risco tem sido demonstrada em quase $100 \%$ de carcinomas cervicais ${ }^{2}$ e foi recentemente mostrado que as infecções persistentes com o mesmo genótipo aumentam fortemente o risco de desenvolvimento de lesões precursoras de alto grau ${ }^{3}$.

Internacionalmente, a sensibilidade de um único teste de Papanicolaou varia de aproximadamente 50 a $60 \%$; assim o sucesso dos programas de rastreio dependem de testes repetidos ${ }^{4}$.

Alguns estudos com base em duplos testes, comparando citologia e teste de DNA-HPV, mostraram maior sensibilidade, mas menor especificidade na detecção de neoplasia intra-epitelial cervical de alto grau de neoplasia ${ }^{5,6}$.

Investigações histológicas das biópsias não encontraram alterações em 23 a $28 \%$ dos casos biopsiados, e que $12-51 \%$ das conizações foram realizadas com base em resultados citológicos falsos positivos ${ }^{7,8}$.

O objetivo deste estudo foi avaliar a acurácia da citologia e colposcopia com resultados histopatológicos em pacientes que foram submetidos à biópsia cervical.

\section{MATERIAIS E MÉTODOS}


Foi realizado um estudo retrospectivo a partir de fevereiro de 2010 a fevereiro de 2012, em um laboratório de referência em patologia cervical. Neste período foram selecionadas 184 mulheres consecutivas que foram submetidas à biópsia cervical.

Exames de congelação foram realizados, seguido pela histologia final. Foram incluídas as mulheres que tinham realizado exames de congelação e que tinham realizado exame de Papanicolaou anterior no mesmo laboratório e no mesmo ano da biopsia cervical. Na maioria dos casos, foi possível acessar os resultados colposcópicos pelo contato com o ginecologista responsável. Preparações histológicas e teste de Papanicolaou foram examinados pelo mesmo patologista e todas as colposcopias foram realizados pelo mesmo ginecologista geral.

Portanto, 184 casos preencheram todos os critérios para análise de acordo com o protocolo do estudo (Exame de Papanicolaou e histologia) e apenas 153 casos realizaram concomitantemente exame de Papanicolaou, colposcopia e histologia.

O teste de citologia utilizado para o rastreio foi desenvolvido por Papanicolau e é conhecido como um exame de Papanicolaou ou citologia oncótica ${ }^{9}$. A amostra é coletada a partir da endocérvice e ectocérvice utilizando espátula e cito-escova, garantindo que as células sejam obtidas a partir de toda a zona de transformação. A amostra é então fixada sobre uma lâmina de vidro, corada com o corante Papanicolaou, e, em seguida, examinada para anomalias cervicais sob a luz do microscópio.

O estudo foi aprovado pelo Comitê de Ética da Universidade do Extremo Sul Catarinense sob o protocolo 350/2011.

\section{Análise estatística}

Os achados de exame de Papanicolaou e achados de exame de colposcopia foram dicotomizadas em baixo grau de lesão intraepitelial escamosa (LIEBG) ou inferior e lesão intraepitelial de alto grau (LIEAG) ou mais.

Além de métodos padrão de estatística descritiva (média, desvio padrão, frequências), os dados foram analisados por tabulação cruzada, o teste do qui- 
Artigo Original

Atenção à Saúde

quadrado, o teste t-Student e estatística de Cohen Kappa, utilizando o software SPSS. Um valor de $\mathrm{p}<0,05$ foi considerado significativo para a avaliação estatística.

A correlação de Spearman foi calculada como uma estimativa da correlação de duas variáveis categóricas de citologia e histologia.

Comparações de duas vias foram usadas para criar tabelas 2x2 e calcular os valores preditivos positivos. A sensibilidade, especificidade, valor preditivo positivo e negativo (VPP, VPN) de cada teste foi calculado e todos eles têm intervalos de confiança binomial exatos de 95\%. O padrão-ouro foi a confirmação histológica.

\section{RESULTADOS}

No período estudado, encontramos 184 pacientes que preencheram os critérios do estudo. A média de idade foi de 30,2 \pm 10,6 (variação 15-89). Um total de $54,3 \%$ das mulheres eram nulíparas, $18,1 \%$ tinham somente um filho e todos as outras eram multíparas. Entre os 184 casos incluídos 153, tiveram resultados de colposcopia (Tabelas 1 e 2).

Tabela 1. Tabela de contingência: Citologia versus histologia.

\begin{tabular}{|c|c|c|c|}
\hline \multirow[b]{2}{*}{ Citologia } & \multicolumn{3}{|l|}{ Histologia } \\
\hline & LIEAG ou mais & LIEBG ou menos & Total \\
\hline LIEAG ou mais & 27 & 12 & 39 \\
\hline IEBG ou menos & 23 & 122 & 145 \\
\hline Total & 50 & 134 & 184 \\
\hline
\end{tabular}

LIEAG-lesão intraepitelial de alto grau; LIEBG- lesão intraepitelial de baixo grau.

Tabela 2. Tabela de contingência: colposcopia versus histologia

\section{Histologia}

\begin{tabular}{cccc}
\cline { 2 - 4 } Colposcopia & LIEAG ou mais & LIEBG ou menos & Total \\
\hline LIEAG ou mais & 31 & 4 & 35 \\
IEBG ou menos & 13 & 105 & 118 \\
\hline Total & 44 & 109 & 153
\end{tabular}

LIEAG-lesão intraepitelial de alto grau; LIEBG- lesão intraepitelial de baixo grau. 
Resultados da citologia: três (1,6\%) tiveram resultados normais, 42 (22,8\%) apresentaram células escamosas de significado indeterminado (ASC-US), 54,3\% apresentaram Lesão Intraepitelial de baixo grau (LIEBG), 20,7\% apresentaram Lesão intraepitelial de alto grau (LIEAG) e um caso de carcinoma invasivo (Tabela 3). Resultados da histopatologia: 68 (37,0\%) casos normais, 6 (3,3\%) ASC-US, 60 $(32,6 \%)$ casos de LIEBG, $42(22,8 \%)$ apresentaram LIEAG e oito $(4,3 \%)$ casos de carcinoma invasivo (Tabela 3 ).

A correlação de Spearman entre os resultados citopatológicos e histopatológicos e resultados colposcópicos e histopatológicos foi a mesma e foi significativa $(r=0,490, p=0,0001)$. O valor Kappa também foi o mesmo para ambos: 0,48 .

A sensibilidade, especificidade e acurácia foram melhores na colposcopia e exame de Papanicolaou: a sensibilidade foi de 70,4\% (IC 95\%, 67,0\% - 81,0\%) e $54,0 \%$ (IC 95\%, 46,8\% - 61,2\%), enquanto que a especificidade foi de $96,3 \%$ (IC 95\% 93,0\% - 99,0\%) e 91,0\% (IC 95\% 86,9\% - 95,1\%) respectivamente. Na citologia o valor preditivo positivo foi de 69,2\% (IC 95\%, 65,5\% - 75,9\%) e valor preditivo negativo de $84,1 \%$ (IC 95\%, 75,3\% - 86,7\%), enquanto na colposcopia o valor preditivo positivo foi de $88,6 \%$ (IC 95\%, 84,0\% - 94,0\%) e valor preditivo negativo foi de $89,0 \%$ (95\% IC 83,7\% - 94,3\%) respectivamente (Tabela 4). A partir dos 42 achados de ASC-US em exame de Papanicolaou, 17 (40,5\%) foram normais na histologia. No entanto, 11 (26,2\%) eram LIEBG, 7 (16,7\%) eram LIEAG e 4 (0,5\%) eram câncer invasivo na histologia (Tabela 3).

Entre os resultados LIEBG no exame de Papanicolaou, 44,0\% foram normais, $41,0 \%$ eram LIEBG e $12,0 \%$ eram LIEAG na histologia. O exame de Papanicolaou e histologia foram concordantes em $60 \%$ dos casos de LIEAG e em $100 \%$ no câncer invasivo (apenas um caso) (Tabela 3). Os resultados colposcópicos foram concordantes com a histologia em $84,4 \%$ dos casos de LIEAG e em $100 \%$ no cancro invasivo (3 casos) (Tabela 5). 
Tabela 3. Correlação de Teste de Papanicolaou e histologia

\begin{tabular}{|c|c|c|c|c|c|c|c|c|c|c|c|c|}
\hline \multirow{3}{*}{ Pap test } & \multicolumn{10}{|c|}{ Histologia } & & \\
\hline & \multicolumn{2}{|c|}{ Normal } & \multicolumn{2}{|c|}{ ASC-US } & \multirow{2}{*}{\multicolumn{2}{|c|}{ LIEBG $\mathbf{n} \%$}} & \multirow{2}{*}{\multicolumn{2}{|c|}{ LIEAG $\mathrm{n} \%$}} & \multicolumn{2}{|c|}{$\begin{array}{c}\text { Câncer } \\
\text { invasivo }\end{array}$} & \multirow{2}{*}{\multicolumn{2}{|c|}{$\begin{array}{c}\text { Total } \\
\text { n \% }\end{array}$}} \\
\hline & \multirow{2}{*}{$\begin{array}{l}\mathbf{n} \\
2\end{array}$} & \multirow{2}{*}{$\begin{array}{l}\% \\
66,7\end{array}$} & \multirow[t]{2}{*}{$\mathbf{n}$} & \multirow[t]{2}{*}{$\%$} & & & & & $\mathbf{n}$ & $\%$ & & \\
\hline Normal & & & & & 1 & 33,3 & - & & - & & 3 & $100,0 \%$ \\
\hline ASC-US & 17 & 40,5 & 3 & 7,1 & 11 & 26,2 & 7 & 16,7 & 4 & 9,5 & 42 & $100,0 \%$ \\
\hline LIEAG & 5 & 13,2 & & & 7 & 18,4 & 23 & 60,5 & 3 & 7,9 & 38 & $100,0 \%$ \\
\hline Câncer invasivo & - & & - & & - & & - & & 1 & 100 & 1 & $100,0 \%$ \\
\hline Total & 68 & 37,0 & 6 & 3,3 & 60 & 32,5 & 42 & 22,8 & 8 & 4,4 & 184 & 100 \\
\hline
\end{tabular}

ASC-US- Atipias de significado indeterminado; LIEAG-lesão intraepitelial de alto grau; LIEBG- lesão intraepitelial de baixo grau.

Tabela 4. Correlação da colposcopia e histologia.

\begin{tabular}{|c|c|c|c|c|c|c|c|c|c|c|c|c|}
\hline \multirow{3}{*}{ Colposcopia } & \multicolumn{10}{|c|}{ Histologia } & & \\
\hline & \multicolumn{2}{|c|}{ Normal } & \multicolumn{2}{|c|}{ ASC-US } & \multicolumn{2}{|c|}{ LIEBG } & \multicolumn{2}{|c|}{ LIEAG } & \multicolumn{2}{|c|}{ Câncer invasivo } & \multicolumn{2}{|c|}{ Total } \\
\hline & $\mathbf{n}$ & $\%$ & $\mathbf{n}$ & $\%$ & $\mathbf{n}$ & $\%$ & $\mathbf{n}$ & $\%$ & $\mathbf{n}$ & $\%$ & $\mathbf{n}$ & $\%$ \\
\hline Normal & 16 & 94.1 & - & & 1 & 5.9 & - & & - & & 17 & $100.0 \%$ \\
\hline ASC-US & & & 1 & 100.0 & & & & & & & 1 & $100.0 \%$ \\
\hline LIEBG & 27 & 27.0 & 5 & 5.0 & 55 & 55.0 & 12 & 12.0 & 1 & 1.0 & 100 & $100.0 \%$ \\
\hline LIEAG & 2 & 6.2 & & & 2 & 6.2 & 27 & 84.4 & 1 & 3.2 & 32 & $100.0 \%$ \\
\hline Câncer invasivo & - & & - & & - & & - & & 3 & 100 & 3 & $100.0 \%$ \\
\hline Total & 45 & 29.4 & 6 & 3.9 & 58 & 37.9 & 39 & 25.5 & 5 & 3.3 & 153 & $100.0 \%$ \\
\hline
\end{tabular}

ASC-US- Atipias de significado indeterminado.LIEAG-lesão intraepitelial de alto grau. LIEBG- lesão intraepitelial de baixo grau. 
Tabela 5. Sensibilidade, especificidade, acurácia, VPP, VPN da colposcopia e citologia comparados com padrão-ouro histologia.

\begin{tabular}{|c|c|c|c|c|c|}
\hline & $\begin{array}{l}\text { Sensibilidade } \\
\text { (IC 95\%) }\end{array}$ & $\begin{array}{l}\text { Especificidade } \\
\text { (IC 95\%) }\end{array}$ & $\begin{array}{l}\text { Acurácia } \\
\text { (IC 95\%) }\end{array}$ & $\begin{array}{c}\text { VPP } \\
\text { (IC 95\%) }\end{array}$ & $\begin{array}{c}\text { VPN } \\
\text { (IC 95\%) }\end{array}$ \\
\hline Colposcopia & $70,4 \%(67,0 \%-81,0)$ & $96,3 \%(93,0 \%-99,0)$ & $88,9 \%(84,0 \%-94.0 \%)$ & $88,6 \%(84,0 \%-94,0 \%)$ & $89,0 \%(83,7 \%-94,3 \%)$ \\
\hline Citologia & $54,0 \%(46,8 \%-61,2 \%)$ & $91,0 \%(86,9 \%-95,1 \%)$ & $80,9 \%(75,3 \%-86,6 \%)$ & $69,2 \%(65,5 \%-75,9 \%)$ & $84,1 \%(75,3 \%-86,7 \%)$ \\
\hline
\end{tabular}

VPP- Valor preditivo positivo. VPN- valor preditivo negativo. 


\section{DISCUSSÃO}

O rastreamento de alta qualidade com a citologia (Papanicolaou) reduziu acentuadamente a mortalidade por câncer do colo do útero de células escamosas, que compreende $80 \%$ a $90 \%$ dos cânceres cervicais ${ }^{10}$.

A correlação da citologia cervical com a análise histológica, embora não seja perfeita, devido a coleta de material e erros na interpretação, permanece como o melhor método de medir a validade do exame de Papanicolaou ${ }^{11}$.

O nosso estudo mostrou que a sensibilidade e especificidade de colposcopia são maiores do que na citologia. Encontramos uma sensibilidade de 54,0\% e especificidade de $91,0 \%$ para Citologia vs Histologia.

Anschau et al. ${ }^{12}$ mostraram que a sensibilidade e especificidade de Citologia foi de $87,3 \%$ e $71,0 \%$, respectivamente. A sensibilidade foi inferior no nosso estudo, enquanto a especificidade foi maior do que os dados da literatura.

Os falsos negativos foram um grande problema detectado em nosso estudo. Devido a isso deixou-se de diagnosticar $46 \%$ dos casos de LIEAG ou mais por citologia e $26 \%$ por colposcopia. Isso realmente é um problema sério porque os exames de Papanicolaou do nosso estudo foram coletados por ginecologistas em seus consultórios e enviados para o mesmo laboratório onde apenas um patologista analisou todos as lâminas, enquanto na saúde pública esta coleta é feita por diferentes profissionais e sua interpretação frequentemente não tem um bom controle de qualidade.

Os exames de colposcopia também foram todos realizados pelo mesmo colposcopista, especialista em ginecologista e em colposcopia. Todos os exames citológicos e de colposcopia foram considerados satisfatórios. A sensibilidade estimada de colposcopia para a detecção de LIEAG 3 variou de $54 \%$ a $85 \%$, conforme documentado em uma meta-análise de estudos 1960-1996 ${ }^{13}$.

Dados precisos sobre a sensibilidade e especificidade do exame de Papanicolaou para lesões pré-neoplásicas são difíceis de obter, mas eles têm sido citados na literatura, variando de $50 \%$ a $90 \%$ e de $31 \%$ para $90 \%$, respectivamente ${ }^{14-18}$. 
Embora o valor preditivo e precisão foi maior em colposcopia do que a citologia, considerando o custo-benefício da citologia, esta é a escolha do teste de triagem, como recomendado pela literatura.

Descobertas interessantes em nosso estudo mostraram "ASC-US" no resultado citológico enquanto no padrão-ouro $16,7 \%$ destes casos foram LIEAG e $0,5 \%$ eram câncer invasivo.

Normalmente, a colposcopia é recomendada para mulheres com LIEBG ou pior na citologia. Nos casos ASC-US a colposcopia é recomendada quando persiste ou está associada com a infecção por HPV de alto risco detectado por testes de DNA $^{19}$. No estudo ATHENA, HPV específico, 16 ou 18 genótipos foram encontrados na NIC 2 ou pior e NIC 3 ou pior em mulheres com ASC-US. O Cobas 4800 HPV teste é clinicamente validado para triagem ASC-US. HPV-16 / HPV-18 genotipagem pode identificar mulheres com maior risco para a doença cervical de alto grau, e essa estratificação de risco adicional pode ser usada na formulação de decisões de gestão paciente ${ }^{20}$. Nossos resultados indicam que seria prudente realizar colposcopia ou HPV genotipagem imediatamente depois de encontrar resultado ASC-US na citologia.

$\mathrm{Na}$ nova diretriz da American Cancer Society os testes de DNA que detectam estirpes de HPV associados ao câncer de colo do útero podem ser usados em conjunto com o teste de Papanicolaou, seja como um teste de rastreio adicional ou quando os resultados do teste de Papanicolaou são incertos ${ }^{21}$.

A estratégia de screening ideal deve identificar os precursores do cancro do colo do útero que possam evoluir para cânceres invasivos e evitar a detecção e tratamento desnecessário de infecção pelo HPV transitória ${ }^{22}$.

No Brasil, a incidência estimada do cancro do colo do útero foi de 52.680 novos casos em 201223. A recomendação atual do Ministério da Saúde no Brasil, para a população em geral, é a realização de citologia anual e após dois resultados negativos consecutivos o exame deve ser realizado a cada três anos ${ }^{24}$. A alta incidência de câncer do colo do útero pode ser devido a falsos negativos e baixa cobertura do exame de Papanicolaou na população de mulheres.

Mais estudos são necessários para analisar a sensibilidade e especificidade da citologia, sendo importante discutir a precisão para detectar lesões precursoras, bem como para um diagnóstico correto do grau das lesões, uma vez que as 
Artigo Original

Atenção à Saúde

diretrizes recomendam o seguimento a partir dos achados do exame de

Papanicolaou.

\section{REFERÊNCIAS}

1. Parkin DM, Bray F.Chapter 2: The burden of HPV- related cancers. Vaccine. $2006 ; 24: 11-25$.

2. Bosch FX, Lorincz A, Munoz N, Meijer CJ, Shah KV. The causal relation between human papillomavirus and cervical cancer. J Clin Pathol. 2002;55:244-65.

3. Kjaer SK, van den Brule AJ, Paull G, Svare EI, Sherman ME, Thomsen BL, et al. Type specific persistence of high-risk human papillomavirus (HPV) as indicator of high grade cervical squamous intraepithelial lesions in young women: population based prospective follow-up study. BMJ 2002;325:(7364):572.

4. Schiffman, M, Castle PE, Jeronimo J, Rodriguez AC, Wacholder S. Human papillomavirus and cervical cancer. Lancet 2007;370:890-907.

5. Ratnam S, Franco EL, Ferenczy A. Human papillomavirus testing for primary screening of cervical cancer precursors. Cancer Epidemiol Biomarkers Prev. 2000;9: $945-51$.

6. Schneider A, Hoyer H, Lotz B, Leistritza S, Kühne-Heid R, Nindl I, et al. Screening for high-grade cervical intra-epithelial neoplasia and cancer by testing for high-risk HPV, routine cytology or colposcopy . Int J Cancer 2000;89:529-34 .

7. Thompson AD, Duggan MA, Nation J, Brasher PM. Investigation of laser cervical cone biopsies negative for pre-malignancy or malignancy. J Low Genit Tract Dis. 2002;6:84-91.

8. Golbang P, Scurry J, de Jong S, McKenzie D, Planner R, Pyman J, et al. Investigation of 100 consecutive negative cone biopsies. $\mathrm{Br} \mathrm{J}$ Obstet Gynaecol. 1997;104:100-4.

9. Papanicolaou GN. A survey of the actualities and potentialities of exfoliated cytology in cancer diagnosis. Ann Intern Med. 1949;31:661-74.

10. Siegel R, Naishadham D, Jemal A. Cancer statistics. CA Cancer J Clin. 2012;62:10-29.

11. Rohr LR. Quality assurance in gynecologic cytology. What is practical? Am J Clin Pathol.1990;94:754-8.

12. Anschau F, Guimarães Gonçalves MA. Discordance between cytology and biopsy histology of the cervix: what to consider and what to do. Acta Cytol. 2011;55:158-62. 
13. Mitchell MF, Schottenfeld D, Tortolero-Luna G, Cantor SB, Richards-Kortum R. Colposcopy for the diagnosis of squamous intraepithelial lesions: a meta-analysis. Obstet Gynecol. 1998;91:626-31.

14. Bernstein SJ, Sanchez-Ramos L, Ndubisi B. Liquid-based cervical cytologic smear study and conventional Papanicolaou smears: a metaanalysis of prospective studies comparing cytologic diagnosis and sample adequacy. Am J Obstet Gynecol. 2001;185:308-17.

15. Arbyn M, Bergeron C, Klinkhamer P, Martin-Hirsch P, Siebers A, Bulten J. Liquid compared with conventional cervical cytology: a systematic review and metaanalysis. Obstet Gynecol. 2008;111:167-77.

16. Cong X, Cox D, Cantor SB. Bayesian meta-analysis of Papanicolaou smear accuracy. Gynecol Oncol. 2007;107:S133-7.

17. Cuzick J, Clavel C, Petry K, Meijer C, Hoyer H, Ratnam S, et al. Overview of the European and North American studies on HPV testing in primary cervical cancer screening. Int J Cancer. 2006;119:1095-101.

18. Koliopoulos G, Arbyn M, Martin-Hirsch P, Kyrgiou M, Prendiville W, Paraskevaidis E. Diagnostic accuracy of human papillomavirus testing in primary cervical screening: a systematic review and metaanalysis of non-randomized studies. Gynecol Oncol. 2007;104:232-46.

19. ASCUS-LSIL Triage Study (ALTS) Group. A randomized trial on the management of low-grade squamous intraepithelial lesion cytology interpretations. Am J Obstet Gynecol. 2003; 188:1393-400.

20. Stoler MH, Wright TC Jr, Sharma A, Apple R, Gutekunst K, Wright TL. ATHENA (Addressing THE Need for Advanced HPV Diagnostics) HPV Study Group. High-risk human papillomavirus testing in women with ASC-US cytology: results from the ATHENA HPV study. Am J Clin Pathol. 2011;135:468-75.

21. American Cancer Society: Cancer Facts and Figures. Atlanta, Ga: American CancerSociety.2012. [Accessed in 25 May] 2012. Available in: http://www.cancer.org/acs/groups/content/@epidemiologysurveilance/documents/doc ument/acspc-031941.pdf.

22. Saslow D, Solomon D, Lawson HW, Killackey M, Kulasingam SL, Cain J et al. American Cancer Society, American Society for Colposcopy and Cervical Pathology, and American Society for Clinical Pathology Screening Guidelines for the Prevention and Early Detection of Cervical Cancer. CA Cancer J Clin. 2012;62:147-72.

23. INCA - Instituto Nacional do Cancer Ministry of Health of Brazil. Incidence of cancer in Brazil. 2012. [Accessed in May 2012]. Available in: http://www1.inca.gov.br/estimativa/2012/estimativa20122111.pdf. 
Artigo Original

Atenção à Saúde

24. INCA- Instituto Nacional de Câncer. MS Brazilian nomenclature for reporting cervical and practices: recommendations for health professionals. J Bras Patol Med Lab. 2006;42:351-73. 